\title{
NUEVAS EVIDENCIAS DE SITIOS ARQUEOLÓGICOS TEHUELCHE/AONIKENK-MAPUCHE (SIGLO XX) EN SANTA CRUZ, PATAGONIA (ARGENTINA)
}

\author{
AMALIA NUEVO-DELAUNAYa ${ }^{a}$ JUAN BAUTISTA BELARDI ${ }^{b} \&$ \\ FLAVIA CARBALLO MARINAc
}

\section{RESUMEN}

El proceso de ocupación estatal del espacio correspondiente a la actual provincia de Santa Cruz hacia finales del siglo XIX involucró su progresiva división en unidades productivas (estancias) y fomentó el establecimiento de grupos criollo-europeos orientados a la cría de ganado ovino. En forma paralela se fueron creando áreas de "reserva" ${ }^{1}$ destinadas al establecimiento de familias de origen indígena. En relación con este proceso se presenta la información correspondiente a cuatro sitios arqueológicos ocupados durante el siglo XX por familias de ascendencia Tehuelche/ Aonikenk-Mapuche. La evidencia material relevada se focaliza sobre estructuras arquitectónicas y raspadores de vidrio. Dos sitios están ubicados en la antigua "reserva" de Lago Viedma ("Cerro Índice"), otro cercano a la misma -en el Hotel Punta del Lago- y, el último, en la Laguna Puesto Peter, lindante con la antigua "reserva" Camusu Aike, en la cuenca media del río Coyle. La nueva información amplía la variabilidad de contextos arqueológicos de tiempos históricos en Patagonia, a la vez que colabora con la visualización de los pueblos originarios.

PALABRAS CLAVE: provincia de Santa Cruz, familias Tehuelche/Aonikenk-Mapuche, estructuras arquitectónicas, raspadores de vidrio.

1 El término "reservas" se indica entre comillas dado que, acorde a la cartografía consultada (Wilkinson \& Lefrancois, 1903; Lefrancois \& Porri, 1927; Herrera,
1926; Jefatura de Policía, 1933-34), estos espacios fueron indistintamente denominados: Reservas, Tribus y/o Concentraciones.

a Centro de Investigación en Ecosistemas de la Patagonia (CIEP), José de Moraleda 16, Coyhaique, Chile. $\bigotimes$ amalia.nuevo@ciep.cl

b Universidad Nacional de la Patagonia Austral, Unidad Académica Río Gallegos, ICASUR, Santa Cruz, Argentina \& Consejo Nacional de Investigaciones Científicas y Técnicas, Argentina. juanbautistabelardi@gmail.com

c Universidad Nacional de la Patagonia Austral, Unidad Académica Río Gallegos, ICASUR, Santa Cruz, Argentina. flaviacarballomarina@gmail.com 


\section{NEW EVIDENCE FOR TEHUELCHE/AONIKENK-MAPUCHE ARCHAEOLOGICAL SITES (XX ${ }^{\mathrm{TH}}$ CENTURY) IN SANTA CRUZ, PATAGONIA (ARGENTINA)}

\section{ABSTRACT}

The process of occupation of Santa Cruz province by the Argentinean State towards the end of the $19^{\text {th }}$ century and during the early $20^{\text {th }}$ century, involved its division in small units (estancias) and promoted the settlement of groups of European descent focused on sheep breeding. At the same time, specific areas ("reservations") were created with the intention of constraining indigenous families. In this vein, we present archaeological evidence of four sites occupied during the $20^{\text {th }}$ century by families of Tehuelche/ Aonikenk-Mapuche origin. The analysis focuses on architectonic features and glass scrapers. Two sites are located inside the former "reservation" area of Lago Viedma ("Cerro Índice"), while the other two sites are placed nearby at the Hotel Punta del Lago and near the former "reservation" of Camusu Aike (Coyle river basin). This evidence broadens the variability of archaeological contexts from Patagonia historical times and advances in the visualization of indigenous people.

KEY WORDS: Santa Cruz Province, Tehuelche/Aonikenk-Mapuche families, architectonic features, glass scrapers.

\section{INTRODUCCIÓN}

Se presenta la evidencia recuperada en cuatro sitios arqueológicos ocupados durante el siglo XX ubicados en el centro-sur de la provincia de Santa Cruz (Fig. 1). Los mismos fueron ocupados por familias de ascendencia Tehuelche/AonikenkMapuche. Los sitios son: Rancho de Zapa, Vega Piaget (ambos con estructuras arquitectónicas y raspadores de vidrio), Hotel Punta del Lago (con raspadores de vidrio) y Laguna Puesto Peter (con raspadores y desechos de vidrio). Según la división catastral provincial, los sitios Rancho de Zapa y Vega Piaget se encuentran dentro del Lote 119 de la Zona Norte del río Santa Cruz, "reserva" Lago Viedma, conocida localmente como "Reserva de Cerro Îndice" o "Vega Piaget" (Rodríguez, 2009). El sitio Hotel Punta del Lago se ubica en la margen este del lago Viedma, dentro del Lote 108 de la Zona Norte del río Santa Cruz, cercano al área anteriormente mencionada. Por último, el sitio Laguna Puesto Peter se encuentra en el área de los Lotes 17/79bis-16/78bis-20/77bis-24/95bis21/76bis de la Zona Sur del río Santa Cruz, lindante a la actual comunidad de Camusu Aike $e^{2}$, sobre el Brazo Norte del río Coyle (cuenca media) (Tabla 1).

El estudio del registro arqueológico se focaliza sobre las diferentes estructuras arquitectónicas relevadas y los raspadores de vidrio (también se reconocieron desechos de talla, de reavivado de filos y fragmentos de botellas). La información presentada amplía la variabilidad de contextos arqueológicos históricos en que se registra la tecnología del vidrio en Patagonia, a la vez que refuerza la visualización histórica de familias de ascendencia Tehuelche/Aonikenk-Mapuche.

Tabla 1. Denominación de los lotes según mapas catastrales de Wilkinson \& Lefrancois (1903), Herrera (1926), Lefrancois \& Porri (1927) y Jefatura de Policía (1933-34).

\begin{tabular}{llll}
\hline Mapa catastral & Rancho de Zapa / Vega Piaget & Hotel Punta del Lago & Laguna Puesto Peter \\
\hline 1903 & Zona no loteada & Zona no loteada & "Tribu Tehuelches" \\
1926 & Desocupado & Ronald Benecke & "Tribu tehuelche" \\
1927 & "Reserva o Concentración Indígena" & Ronaldo Benecke & "Tribu tehuelches" \\
$1933-34$ & "Concentración de Indígenas" & Indeterminada & "Reserva Tehuelche" \\
\hline
\end{tabular}

2 Desde 2007, año en que es reconocida la personería jurídica de Camusu Aike, sus residentes no utilizan el término "reserva" sino comunidad (www.camusuaike.com) (Rodríguez, 2008, p.15). 


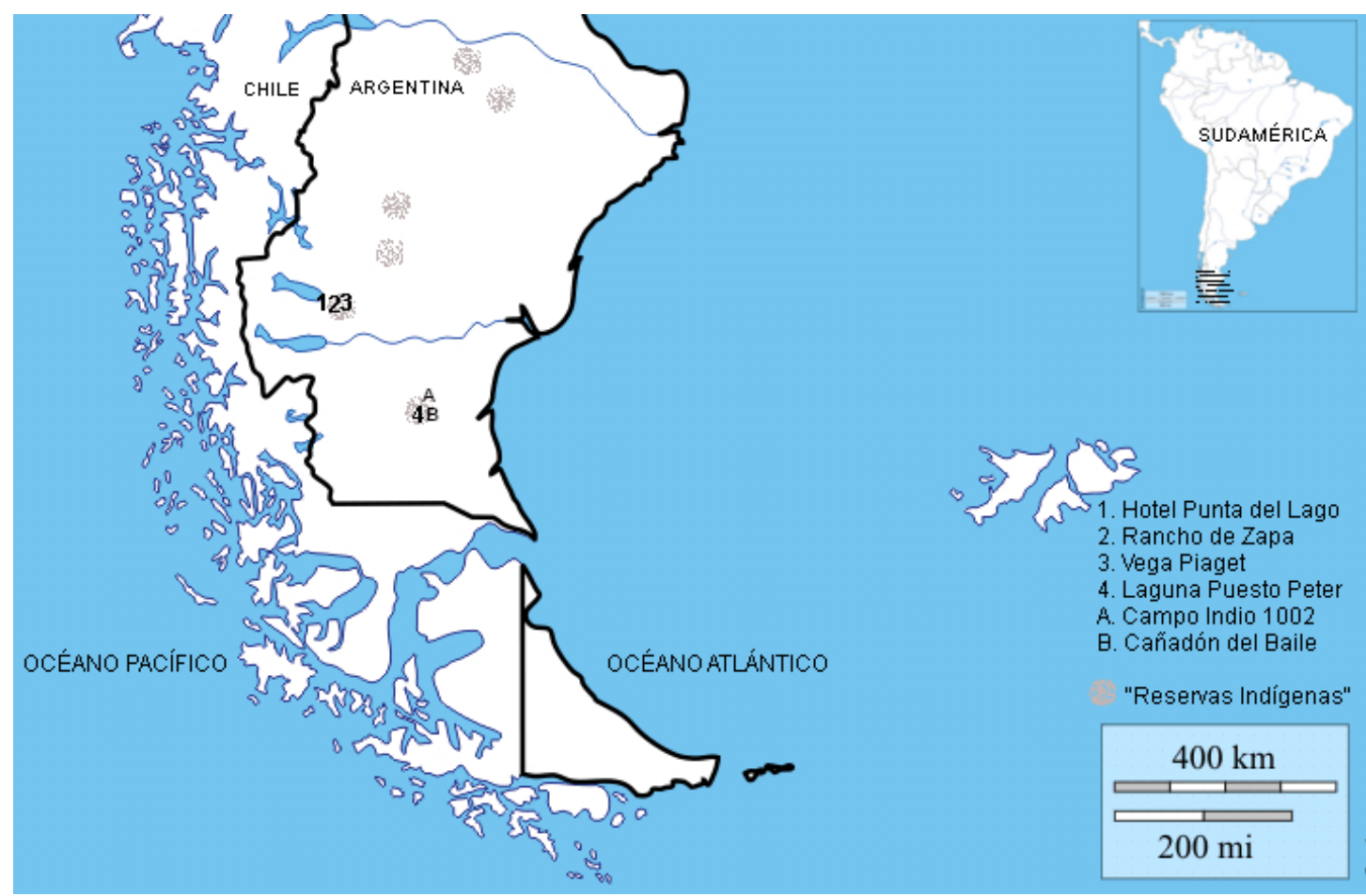

Fig. 1. Ubicación de los sitios mencionados en el texto y de los espacios de "reservas" en el Territorio de Santa Cruz hacia principios del siglo XX (en paréntesis año de creación y nombre de actual comunidad territorial). De Sur a Norte: Camusu Aike (1898, comunidad territorial Camusu Aike), Lago Viedma (1922), Lago Cardiel Lote $28 b i s$ (1922, comunidad territorial Lote 28 bis), Lago Cardiel Lote 6 (1922), Paraje Villa Picardo -Las Heras parte de los lotes 2 y 9- (1926, comunidad territorial Millaqueo) y Laguna Sirven -Las Heras parte de los lotes 21 a-b y 22 a-b-d fracción C- (1927, comunidad territorial Limonao) (Barbería, 1996; Enrici et al. 2006; Rodríguez, 2008, 2009, 2010; Rodríguez \& Delrío, 2000; Rodríguez \& Horlent, 2016).

\section{METODOLOGÍA}

Los sitios arqueológicos fueron reconocidos durante tres campañas (marzo, septiembre y noviembre) llevadas a cabo en el año 2015. Para el relevamiento de las estructuras se siguieron los lineamientos definidos por Nuevo Delaunay (2016). En primer lugar, se las individualizó y georreferenció, luego se registraron las características de cada una de ellas según sus dimensiones, los métodos de construcción (técnicas y materiales usados para su edificación) y el estado de conservación: alto, medio y bajo (medida relativa que se obtiene en función del estado en que se encuentran las estructuras en un sitio y sobre el total de las presentes en los distintos sitios). El uso dado a las estructuras fue inferido sobre la base de sus morfologías y artefactos asociados. La extensión de cada sitio se definió tomando en consideración el perímetro de dispersión de estructuras y materiales presentes.
Además, se definió la presencia de zonas de descarte (áreas donde se detectó la mayor frecuencia de restos arqueológicos), en las que se plantearon unidades de recolección superficial (U.R.) de dimensiones variables. En el sitio Rancho de Zapa se trazaron cinco unidades (U.R. 1 a 5) de $3 \mathrm{~m}^{2}$ cada una, mientras que en Vega Piaget se distinguieron seis unidades (U.R. 1 a 6) de entre 0,5 y $4 \mathrm{~m}^{2}$. En ambos también se reconocieron materiales fuera de las U.R. Dado que los sitios Hotel Punta del Lago y Laguna Puesto Peter presentaron una menor frecuencia artefactual se planteó una sola unidad donde se recuperaron todos los elementos.

Para el análisis de los raspadores vítreos se utilizó la tipología propuesta por C. Aschero (1975, 1983), dado que sus características tecnotipológicas permiten describirlos empleando las mismas variables que las de los artefactos líticos. Se determinó la forma base o sección utilizada de envases (boca, cuello, hombro, cuerpo y base [Rock, 1990]) y se los 
discriminó por color (verde, marrón, transparente). También se registró su tipo, procedencia y cronología relativa a partir de la identificación de elementos diagnósticos como bases y golletes (e.g. Jones \& Sullivan, 1989; Kendrick, 1963, 1967, 1968).

\section{LOS SITIOS}

Rancho de Zapa

El sitio Rancho de Zapa (4950'2.40"S; 71.44'25.84"O, $514 \mathrm{msnm}$ ) se ubica a $20 \mathrm{~km}$ al sureste del lago Viedma y de la Ruta Nacional $\mathrm{N}^{\circ} 40$ y al pie del Cerro Índice (Fig. 1).

Según estudios etnohistóricos y antropológicos este espacio habría sido ocupado por familias de ascendencia mapuche-tehuelche y tehuelche (Rodríguez, 2009, p. 4) durante principios y hasta mediados del siglo XX, entre las que se encontraba la familia de Juan Pascual (Priegue, 2007, p. 25) y la familia de Luisa Mercerat y Ángel Zapa (Halvorsen, 2011, pp. 209-221).
El sitio ocupa 2,6 ha (Fig. 2) y se ubica sobre un mallín que desemboca en la margen norte del arroyo Los Paisanos. Se identificaron 10 estructuras arquitectónicas y un pozo, que dan cuenta de una amplia variabilidad de tipos, formas, dimensiones, métodos de construcción y estados de conservación (Fig. 3a y b).

La estructura \#1 (8,3 m x 6,2 m) es de planta rectangular con uso de piedra canteada seca en los cimientos y por encima ladrillos de adobe. El piso es de tierra y no se conservan restos del techo. No presenta divisiones internas y el estado de conservación es bajo. La estructura se interpreta como de tipo habitacional.

La estructura \#2 (1,4 m x 1,2 m) es un baño. Los cimientos son de cemento y se han usado vigas de madera para el techo y chapa corrugada para las paredes. El piso es de cemento y baldosas. $\mathrm{Su}$ estado de conservación es medio.

En la estructura \#3 (14 m x 10,2 m) se mantienen solo los cimientos de cemento $y$
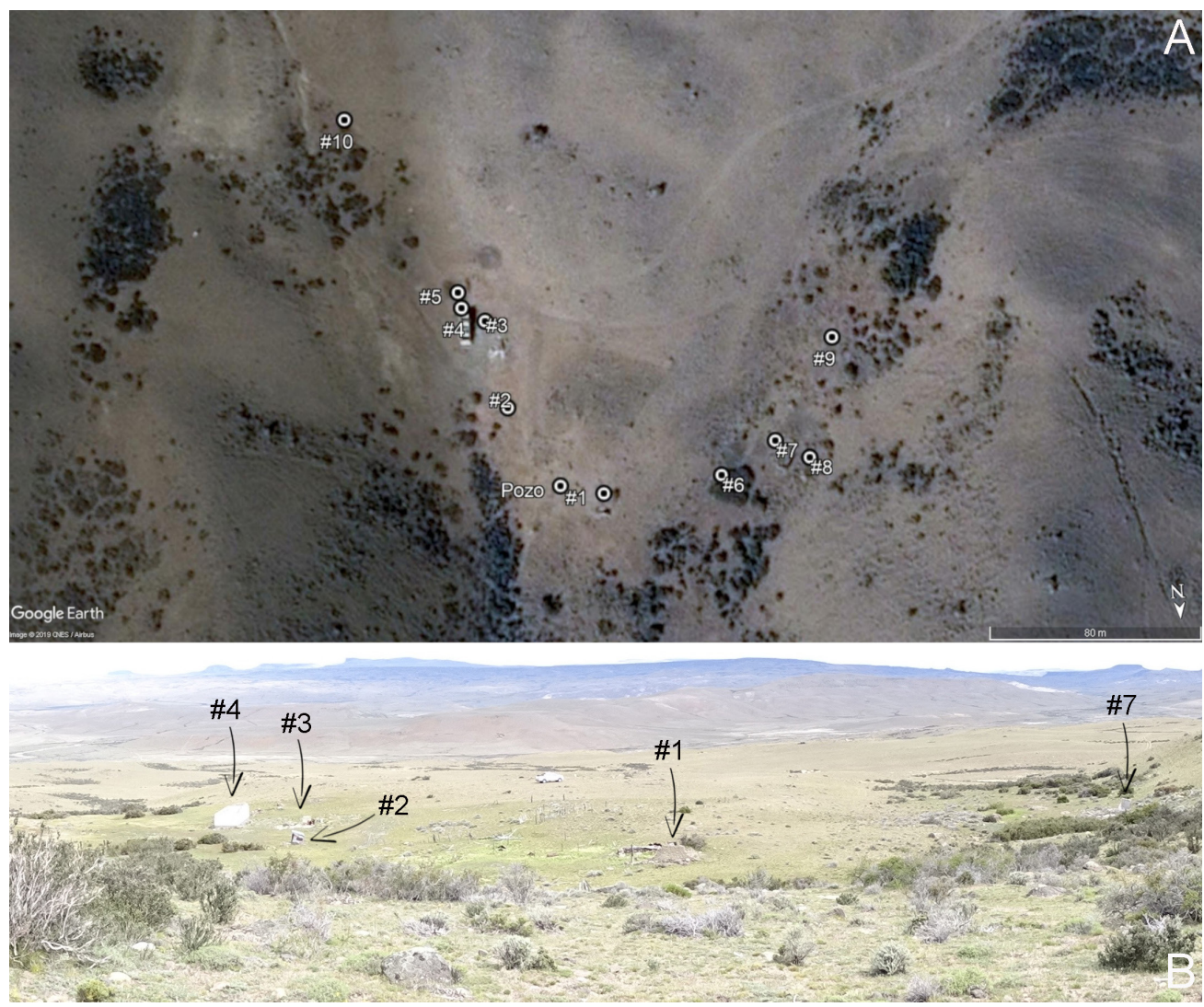

Fig. 2. Vistas generales del sitio Rancho de Zapa: (A) vista satelital y ubicación de estructuras y pozo, (B) vista general en dirección SO (se identifican en la imagen las estructuras \#1, \#2, \#3, \#4 y \#7). 
algunas baldosas pertenecientes al piso. Su estado de conservación es bajo y su función no pudo ser determinada.

La estructura \#4 (10,3 m x 3,5 m) fue construida con ladrillos y bloques de cemento unidos con argamasa. Presenta revoque fino de cemento en las paredes y el piso es de cemento alisado. Tiene tres divisiones internas, una abertura de tipo puerta y tres tipo ventanas. Aunque no se conservan restos del techo, es posible observar que era de tipo "a un agua". El estado de conservación es medio. La estructura se interpreta como habitacional.
La estructura \#5 está compuesta por distintas secciones construidas de piedra seca sin cantear. Una primera sección en forma de "L" (9,1 m x 5 $\mathrm{m})$, correspondiente a los cimientos; una segunda de forma cuadrangular (4,1 $\mathrm{m} \mathrm{x} \mathrm{3,3} \mathrm{m}$ ) a la que se le ha colocado una especie de puerta con orientación Este; y una tercera en forma de " $U$ " (4,1 m de boca x 5,9 m de profundidad) que pareciera haber sido (re)utilizada como depósito de cenizas. Su estado de conservación es bajo. Su función no pudo ser determinada.

La estructura \#6 (1,5 $\mathrm{m} \times 1,3 \mathrm{~m})$ es un baño de madera y chapa corrugada. El estado de conservación es bajo.

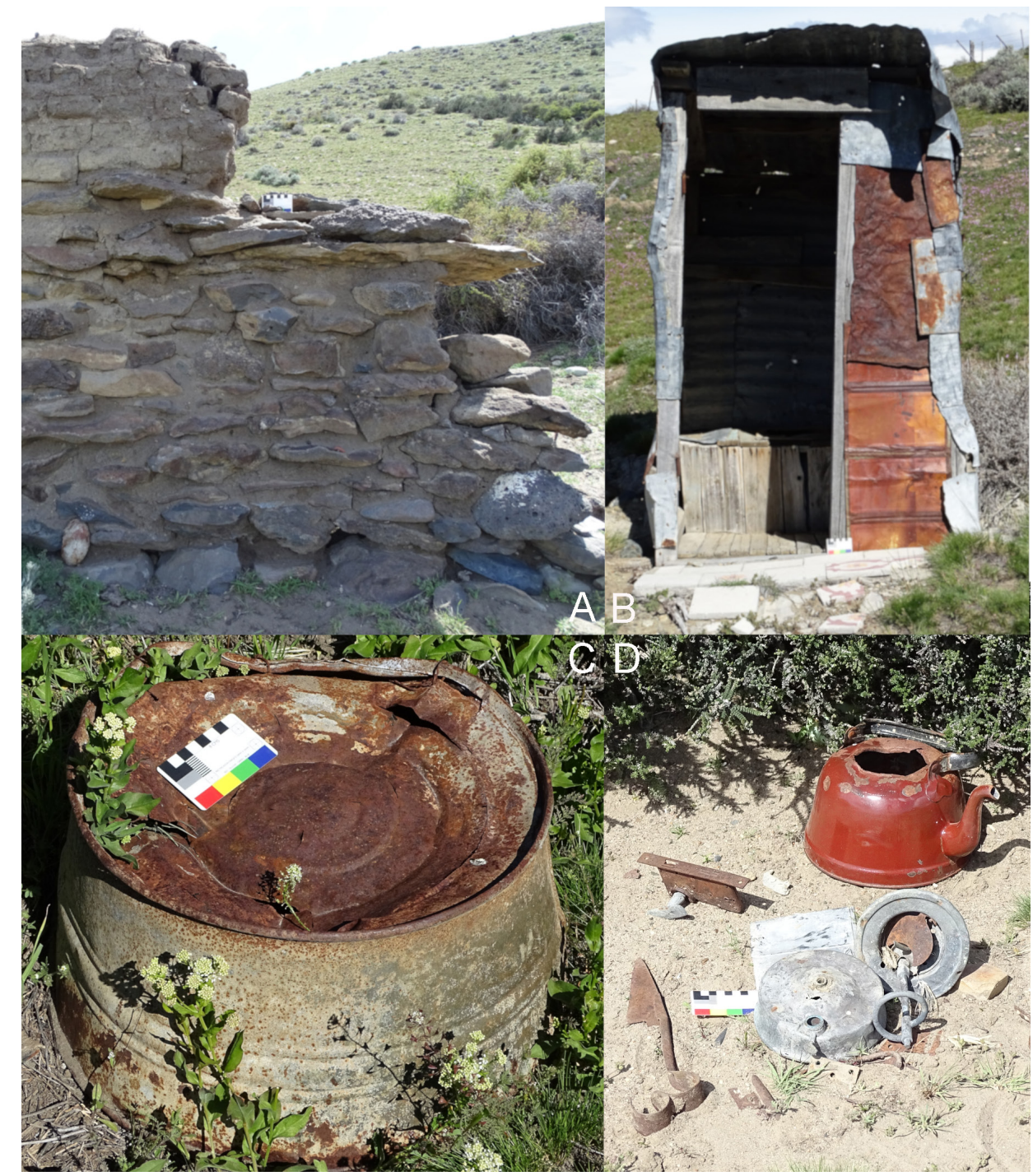

Fig. 3. Estructuras y materiales en el sitio Rancho de Zapa: (A) detalle estructura \#1, (B) estructura \#2, (C) Palangana y (D) Tijeras de esquilar, cerraduras y pava. 
La estructura \#7 (8,2 m x 4,3 m) está construida con ladrillos industriales con cemento como argamasa, sin divisiones internas. Presenta revoque fino de cemento en las paredes y no conserva restos de techo. El estado de conservación es bajo. La estructura se interpreta como de tipo habitacional.

La estructura \#8 (5,2 m x 2,9 m x $5 \mathrm{~m}$ $\mathrm{x} 4,7 \mathrm{~m}$ ) es de forma trapezoidal y construida con piedra seca sin cantear. Su estado de conservación es bajo. Dada la presencia de una chimenea en su interior se interpreta como de tipo habitacional.

La estructura \#9 (9,5 m x 5 m) está construida con piedra seca sin cantear en sus cimientos $y$, por encima, piedras laja y ladrillos de adobe con uso de argamasa. No tiene techo. Su estado de conservación es bajo y se interpreta como de tipo habitacional.

La estructura \#10 (5,2 m x 4,6 m x 3,9 m x $4,9 \mathrm{~m}$ ) fue construida con piedra canteada sin uso de mortero. Su estado de conservación es bajo y su función no pudo ser determinada.

Además, se identificó un pozo excavado (9 m x $1 \mathrm{~m}$ y $20 \mathrm{~cm}$ de profundidad) que podría vincularse con el manejo de agua.

Los artefactos registrados en asociación con las estructuras son diversos y corresponden a elementos de la vida cotidiana rural, entre los que observamos materiales de construcción como ladrillos y chapas $(\mathrm{N}=20)$, utensilios de cocina $y$ mobiliario doméstico $(\mathrm{N}=19)$, contenedores de alimentos y bebidas $(\mathrm{N}=16)$ y herramientas para el manejo de animales ( $\mathrm{N}=2$ ) (Fig. 3c y d). Se asocian en cada caso con las funciones inferidas para las estructuras (e.g. en las habitaciones se encuentran utensilios de cocina, mientras que en el pozo excavado hay contenedores y herramientas para el manejo de animales $y$ vegetales). A los distintos materiales, en su mayoría se les reconoce algún tipo de reciclaje o ciclaje lateral (sensu Schiffer, 1990; Skibo \& Schiffer, 2001). Por último, en las U.R. se observaron concentraciones de material vítreo, mayoritariamente raspadores $(\mathrm{N}=52)$, sumados a restos óseos de fauna muy fragmentados y quemados. Las cinco U.R. definidas se encuentran dispersas en distintos sectores del sitio. En todos los casos se sitúan lindantes con plantas de molle
(Schinus sp.), con presencia de los materiales en superficie que, en algunos casos, no fue posible recuperar por encontrarse muy cubiertos por sus ramas.

\section{Vega Piaget}

El sitio Vega Piaget (4949'22.95"S; $71^{\circ} 42^{\prime} 14.64^{\prime \prime O}$ ) se ubica a $426 \mathrm{msnm}$ y a tres $\mathrm{km}$ al oeste de Rancho de Zapa. También se sitúa sobre un mallín que desemboca en la margen norte del arroyo Los Paisanos. Este espacio habría sido ocupado por las mismas familias de ascendencia mapuche-tehuelche y tehuelche y durante la misma época que el sitio Rancho de Zapa (Priegue, 2007, p. 25; Rodríguez, 2009, p. 4; Halvorsen, 2011, pp. 209-221).

El sitio se extiende a lo largo de 0,94 ha. Se reconocieron tres $(\mathrm{N}=3)$ estructuras, cuatro $(\mathrm{N}=4)$ pozos para el manejo de agua y un espacio utilizado como huerta (Fig. 4a y b).

Para la construcción de la estructura \#1 (7 m $x 4 \mathrm{~m}$ ) se utilizaron vigas de madera y chapa para el techo y las paredes. El techo es "a un agua". Se registran dos aberturas: una ventana y una puerta. Su estado de conservación es alto y se interpreta como de tipo habitacional.

La estructura \#2 (3,4 m x 1,4 m) es de tipo rectangular y corresponde a cimientos de piedra seca sin cantear. El estado de conservación es bajo y su función no pudo ser determinada.

La estructura \#3 (15 m de diámetro y 1,3 m de altura) es un corral circular de maderas y alambres con un palenque de madera en el centro (posiblemente relacionado con el manejo de caballos -Equus caballus). Su estado de conservación es medio.

Los pozos identificados \#1 (1 $\mathrm{m} \times 1 \mathrm{~m}), \# 2$ (6 m x 3,2 m), \#3 (8 m x 2,5 m) y \#4 (1 m x $1 \mathrm{~m})$ habrían sido empleados para el manejo de agua. En el caso del \#1 y \#4, son cuadrangulares; el \#2, es una excavación rectangular cercana a un sauce (Salix humboldtiana) y el \#3 es rectangular y se ubica cerca de una escorrentía.

Un espacio de $24 \mathrm{~m} \times 9 \mathrm{~m}$, rodeado de sauces y con plantas de grosellas (Ribes rubrum) en su extremo sureste, fue reconocido como huerta.

Los artefactos asociados a las estructuras son escasos $(\mathrm{N}=9)$ y fueron relevados principalmente en la \#1 (Fig. 4c y d). En su mayoría presentan algún tipo de reciclaje o ciclaje lateral (sensu 


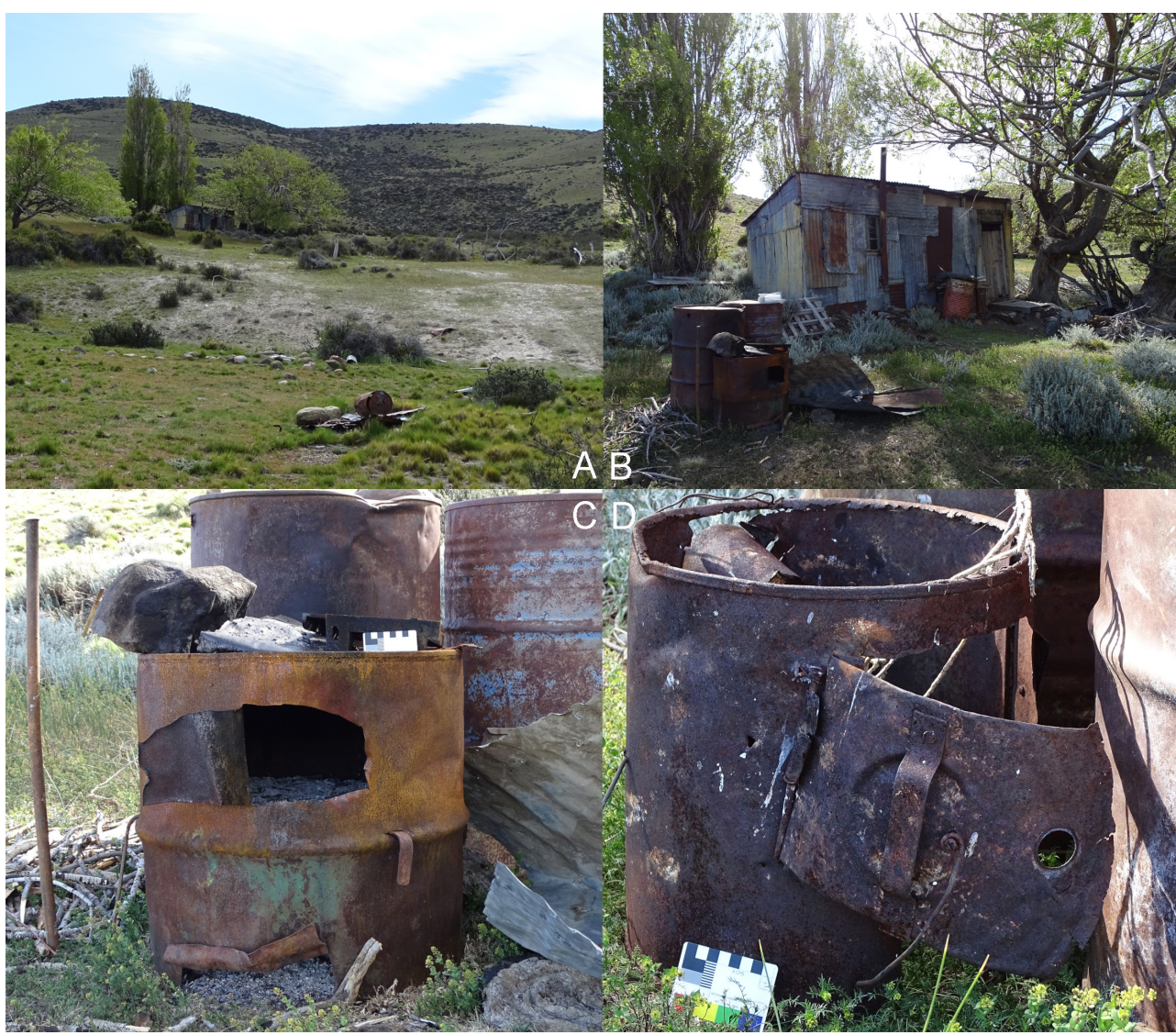

Fig. 4. Estructuras y materiales en sitio Vega Piaget: (A) vista general,

(B) estructura \#1, (C) Parrilla y (D) Cocina.

Schiffer, 1990; Skibo \& Schiffer, 2001). En las U.R. se registró principalmente material vítreo, con predominio de raspadores $(\mathrm{N}=37)$, sumado a restos faunísticos muy fragmentados y quemados. Las seis U.R. definidas, a diferencia del sitio Rancho de Zapa, son de tamaño variable y se sitúan todas por detrás de la estructura \#1. Además, solo en cuatro casos lindan con plantas de molle, mientras que los dos restantes se encuentran sin vegetación circundante. En todas las U.R. los artefactos se encontraron en superficie.

\section{Hotel Punta del Lago}

El sitio Hotel Punta del Lago (4947’06.24"S; 72॰03'59.06"O) se corresponde con el basural de un antiguo hotel de campaña. Se ubica a 251 msnm, sobre la margen este del lago Viedma, en la estancia Punta del Lago. El área del sitio ocupa aproximadamente 0,1 ha en la que se registró un gran basural lindante con una laguna (Fig. 5a). Se reconocieron dos raspadores de vidrio.

\section{Laguna Puesto Peter}

El sitio Laguna Puesto Peter (5057'38.50"S; 7046'49.40"O) se localiza a $243 \mathrm{msnm}$, sobre la margen SO de la laguna Puesto Peter, conectada con la margen norte del Brazo Norte del río Coyle (cuenca media), y relacionado espacialmente con el área de la antigua "reserva" de Camusu Aike (Belardi et al. 2013). El área ocupada es de 0,3 ha en la que se recuperaron tres raspadores y fragmentos vítreos en superficie (Fig. 5b), junto con fragmentos óseos. No se registraron estructuras.

\section{RASPADORES DE VIDRIO}

El conjunto de raspadores está compuesto por 94 ejemplares (Fig. 6; Tabla 2). Alrededor del 


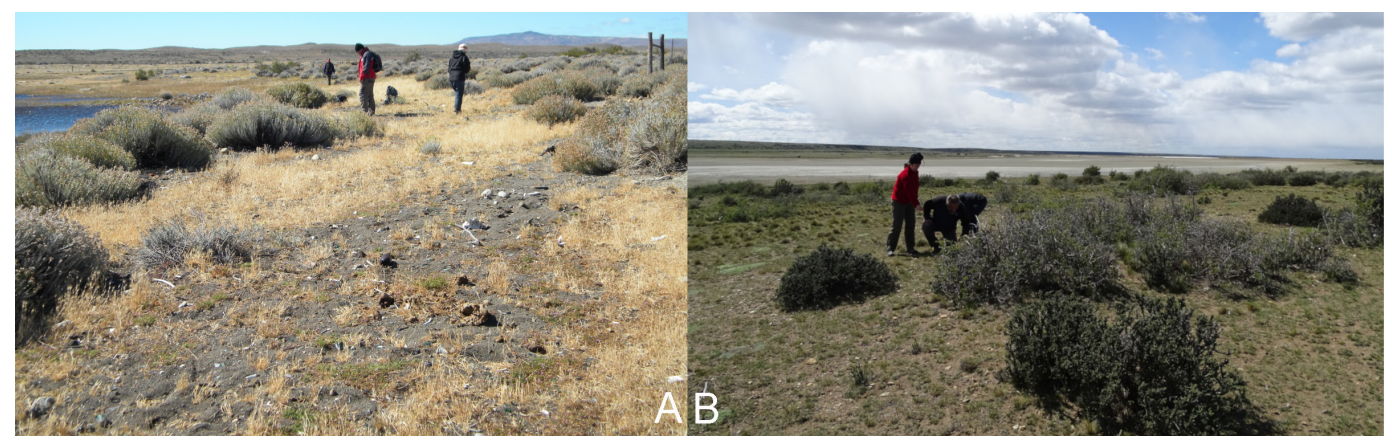

Fig. 5. Vista general: (A) sitio Hotel Punta del Lago y (B) sitio Laguna Puesto Peter.

$65 \%$ se encuentra entero o presenta fracturas que no interfieren con la adscripción a un subgrupo tecnotipológico.

En un único caso se usó vidrio de ventana para la manufactura de un raspador (sitio Vega Piaget), el resto fue confeccionado sobre el cuerpo de contenedores. Esto podría deberse a que es la porción que exhibe mayor superficie para la extracción de preformas. Se han empleado fragmentos transversales a su eje longitudinal, aunque también se reconocen otras secciones de botellas y frascos como hombros y bases. Dado que los raspadores están hechos en segmentos muy reducidos de contenedores, no se puede identificar el tipo empleado, por lo que en la mayoría de los casos no fue posible atribuir cronologías de fabricación. En 24 casos (Rancho de Zapa N=12 y Vega Piaget $\mathrm{N}=12$ ) pudieron reconocerse "marcas de unión de tipo industrial", una característica presente en botellas manufacturadas en serie desde comienzos del siglo XX. El único gollete recuperado corresponde a uno de tipo double ring aplicado, el que provendría de botellas de molde sopladas. Las mismas dejaron de producirse hacia finales del siglo XIX (Kendrick, 1967; Jones \& Sullivan, 1989).

La coloración de los raspadores está dominada por el verde $(90,3 \%)$ en sus distintas tonalidades -que fue usado en botellas de todo tipo y adscripción temporal-, aunque también se registran otros sobre vidrio marrón (5,4\%) y transparente (4,3\%). Según la ubicación de sus filos, el $76,6 \%$ es de filo perimetral seguido por el fronto-lateral $(3,2 \%)$ y el frontal $(3,2 \%)$. El $17 \%$ restante no pudo determinarse (Tabla 2$)$. Las dimensiones de los raspadores (Tablas 3, 4 y 5) no sugieren una estandarización en la manufactura, indicando posiblemente la reactivación de los filos.

Tabla 2. Frecuencias y porcentajes de raspadores según la ubicación del filo.

\begin{tabular}{lccccc}
\hline Ubicación del filo & $\begin{array}{c}\text { Rancho de Zapa } \\
(\mathrm{N} / \%)\end{array}$ & $\begin{array}{c}\text { Vega Piaget } \\
(\mathrm{N} / \%)\end{array}$ & $\begin{array}{c}\text { Hotel Punta del Lago } \\
(\mathrm{N} / \%)\end{array}$ & $\begin{array}{c}\text { Laguna Puesto Peter } \\
(\mathrm{N} / \%)\end{array}$ & $\begin{array}{c}\text { Total } \\
(\mathrm{N} / \%)\end{array}$ \\
\hline PERIMETRAL & $42 / 44,7$ & $27 / 28,7$ & $1 / 1,1$ & $2 / 2,1$ & $72 / 76,6$ \\
FRONTO-LATERAL & --- & $2 / 2,1$ & --- & $1 / 1,1$ & $3 / 3,2$ \\
FRONTAL & -- & $2 / 2,1$ & $1 / 1,1$ & -- & $3 / 3,2$ \\
INDETERMINADO & $10 / 10,6$ & $6 / 6,4$ & --- & --- & $16 / 17,0$ \\
\hline Total & $52 / 55,3$ & $37 / 39,4$ & $2 / 2,1$ & $3 / 3,2$ & $94 / 100$ \\
\hline
\end{tabular}

Tabla 3. Medidas del largo de los raspadores.

\begin{tabular}{lcccc}
\hline \multicolumn{1}{c}{ Sitio } & $\begin{array}{c}\text { Largo máximo } \\
(\mathrm{cm})\end{array}$ & $\begin{array}{c}\text { Largo mínimo } \\
(\mathrm{cm})\end{array}$ & $\begin{array}{c}\text { Promedio } \\
(\mathrm{cm})\end{array}$ & Desviación estándar \\
\hline Rancho de Zapa & 5,2 & 3 & 4,1 & 1,5556349 \\
Vega Piaget & 5,6 & 2 & 3,8 & 2,5455844 \\
Hotel Punta del Lago & 4,0 & 3,7 & 3,9 & 0,2121320 \\
Laguna Puesto Peter & 4,4 & 3,9 & 4,15 & 0,3535534 \\
\hline
\end{tabular}


Tabla 4. Medidas del ancho de los raspadores.

\begin{tabular}{lcccc}
\hline \multicolumn{1}{c}{ Sitio } & $\begin{array}{c}\text { Ancho máximo } \\
(\mathrm{cm})\end{array}$ & $\begin{array}{c}\text { Ancho mínimo } \\
(\mathrm{cm})\end{array}$ & $\begin{array}{c}\text { Promedio } \\
(\mathrm{cm})\end{array}$ & Desviación estándar \\
\hline Rancho de Zapa & 6,9 & 3,8 & 5,4 & 2,192031 \\
Vega Piaget & 5,5 & 3,5 & 4,5 & 1,4142136 \\
Hotel Punta del Lago & 3,2 & 2,7 & 3,0 & 0,3535533 \\
Laguna Puesto Peter & 4,9 & 4,3 & 4,6 & 0,4242641 \\
\hline
\end{tabular}

Tabla 5. Medidas del espesor de los raspadores.

\begin{tabular}{lcccc}
\hline \multicolumn{1}{c}{ Sitio } & $\begin{array}{c}\text { Espesor máximo } \\
(\mathrm{cm})\end{array}$ & $\begin{array}{c}\text { Espesor mínimo } \\
(\mathrm{cm})\end{array}$ & $\begin{array}{c}\text { Promedio } \\
(\mathrm{cm})\end{array}$ & $\begin{array}{c}\text { Desviación } \\
\text { estándar }\end{array}$ \\
\hline Rancho de Zapa & 0,6 & 0,3 & 0,45 & 0,212132 \\
Vega Piaget & 0,6 & 0,3 & 0,45 & 0,212132 \\
Hotel Punta del Lago & 0,3 & 0,3 & 0,3 & 0 \\
Laguna Puesto Peter & 0,6 & 0,6 & 0,6 & 0 \\
\hline
\end{tabular}

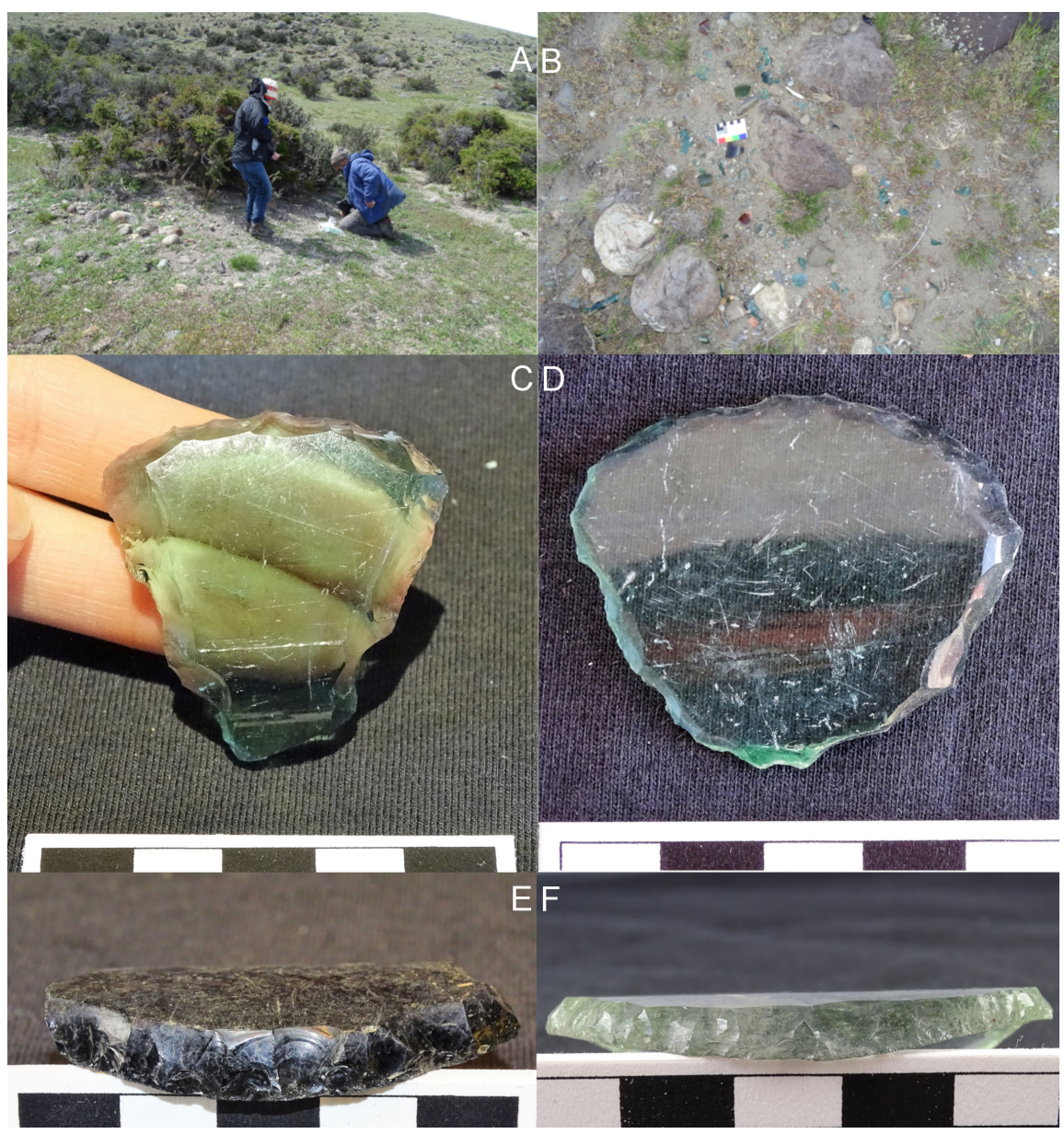

Fig. 6. Raspadores de vidrio: (A) U.R. 1 en sitio Rancho de Zapa, (B) U.R. 6 en sitio Vega Piaget, (C y F) sitio Vega Piaget, (D) sitio Rancho de Zapa, y (E) sitio Laguna Puesto Peter. 


\section{COMENTARIOS FINALES}

El proceso de ocupación estatal del espacio correspondiente a la actual provincia de Santa Cruz (finales del siglo XIX), involucró su progresiva división en unidades productivas (estancias) y fomentó el establecimiento de grupos criollo-europeos orientados a la cría de ganado ovino. El mismo incluyó, en forma paralela, la creación de áreas destinadas al establecimiento de familias de origen indígena ("reservas") (Barbería, 1987, 1996; Bandieri, 2005). El registro de estructuras arquitectónicas y raspadores de vidrio en los sitios arqueológicos ocupados durante el siglo XX por familias de ascendencia Tehuelche/ Aonikenk-Mapuche recién descritos se suma a los recuperados en otros sitios contemporáneos (aunque no necesariamente sincrónicos) de Patagonia (Nuevo Delaunay et al. 2017). La presencia de raspadores de vidrio y de gres cerámico da cuenta del trabajo del cuero, relacionado con la confección de quillangos y la venta de pieles principalmente de guanaco (Lama guanicoe) desde fines del siglo XIX, una actividad comercial frecuente en familias de ascendencia Tehuelche/Aonikenk-Mapuche (e.g. Gómez Otero, 1987; Aguerre, 2000; Priegue, 2007; Belardi et al. 2013; Nuevo Delaunay et al. 2017). Entre estos distintos sitios se destacan, por un lado, aquellos de ocupaciones de áreas marginales -en relación con la accesibilidad y en función de intereses criollo-ganaderos-, ubicados por fuera de las áreas de antiguas "reservas" (Nuevo Delaunay, 2012, 2016) y, por otro, los que presentan ocupaciones al interior de éstas, como en el caso de Camusu Aike. En ella se propuso el uso de toldos en los sitios Cañadón del Baile (Carballo Marina et al. 2000-2002) y Campo Indio 1002 (Belardi et al. 2013). Además, existen estructuras habitacionales que conforman el actual núcleo poblacional de la comunidad que se encuentra sobre el cañadón Camusu Aike.

Otros sitios ubicados en contextos de antiguas "reservas" -tales como los ahora presentados Rancho de Zapa y Vega Piagetdan cuenta de variados tipos de estructuras arquitectónicas, métodos y materiales de construcción y áreas con altas frecuencias de raspadores de vidrio, lo que estaría relacionado con una permanencia de largo plazo, acorde con el registro etnohistórico (Rodríguez, 2009; Priegue, 2007; Halvorsen, 2011). Por su parte, los sitios ubicados en contextos por fuera de antiguas "reservas" pero cercanos o lindantes a las mismas -como Hotel Punta del Lago y Laguna Puesto Peter-, presentan bajas frecuencias de raspadores. Los hallazgos de Hotel Punta del Lago fueron realizados en un basural del hotel y podrían ser el resultado del descarte en contextos de uso circunstancial (por ej. trabajos "por día y/o conchavo"), mientras que Laguna Puesto Peter correspondería a un campamento transitorio o de paso. Este último caso es interesante en cuanto amplía la variabilidad de tipos de sitios conocidos en la antigua "reserva" de Camusu Aike. Así, sobre la base de la superficie y la frecuencia de raspadores, se diferencia de los sitios Cañadón del Baile y Campo Indio 1002 que se interpretaron como campamentos residenciales, con mayor intensidad de uso en el último caso (Belardi et al. 2013).

La información generada desde la arqueología se suma a aquella proveniente de trabajos etnohistóricos y antropológicos, enriqueciendo el conocimiento respecto de grupos y familias de ascendencia Tehuelche/AonikenkMapuche en Patagonia durante el siglo XX y colaborando con su visualización histórica.

\section{AGRADECIMIENTOS}

Al Sr. Sergio Raggi (Ea. La Margarita) y a la Sra. Ana Rojo (Ea. Punta del Lago) por su inestimable ayuda durante los trabajos de campo. A Anahí Re por su apoyo en terreno y a César Méndez por su colaboración con las figuras. A los dos evaluadores anónimos por sus valiosos comentarios.

A la Unidad Académica Río Gallegos de la Universidad Nacional de la Patagonia Austral y al Programa Regional ANID R17A10002.

\section{BIBLIOGRAFÍA}

Aguerre, A. M. (2000). Las vidas de Pati en la toldería tehuelche del Río Pinturas y el después. Buenos Aires: Universidad de Buenos Aires.

Aschero, C. (1975). Ensayo para una clasificación morfológica 
de los instrumentos líticos aplicada a estudios tipológicocomparativos. Buenos Aires, Consejo Nacional de Investigaciones Científicas y Técnicas, Ms.

Aschero, C. (1983). Revisión del ensayo para una clasificación morfológica de los instrumentos líticos aplicada a estudios tipológico-comparativos. Buenos Aires, Consejo Nacional de Investigaciones Científicas y Técnicas, Ms.

Bandieri, S. (2005). Historia de la Patagonia. Buenos Aires: Sudamericana.

Barbería, E. M. (1987). El avance de la ganadería ovina y el indígena de Santa Cruz. Mundo Ameghiniano, 7, 1953.

Barbería, E. M. (1996). Los dueños de la tierra en la Patagonia Austral, 1880-1920. Santa Cruz: Universidad Nacional de la Patagonia Austral.

Belardi, J. B., Carballo Marina, F., Nuevo Delaunay, A., \& De Angelis, H. (2013). Raspadores de vidrio y gres cerámico en la reserva tehuelche (Aonikenk) de Camusu Aike: aportes al conocimiento de poblaciones indígenas de los siglos XIX y XX en el territorio de Santa Cruz. Relaciones, 38(1), 37-57.

Carballo Marina, F., Belardi, J. B., Espinosa, S., \& Ercolano, B. (2000-2002). Tecnología y movilidad en el río Coyle, Santa Cruz. Cuadernos del Instituto Nacional de Antropología y Pensamiento Latinoamericano, 19, 89-107.

Enrici, A., Álvarez, P., Yung, P., \& Lillevangstu, E. (2006). La cultura después de la Cultura. Estudio de la dinámica en la minoría tehuelche Aónikenk, en proceso de extinción. Reserva Indígena de Camusu Aike, provincia de Santa Cruz. En J. B. Belardi, F. Carballo Marina \& S. Espinosa (Eds.), La cuenca del río Coyle. Estado actual de las investigaciones (pp. 173-190). Santa Cruz: Universidad Nacional de la Patagonia Austral.

Gómez Otero, J. (1987). Un raspador en vidrio confeccionado por una tehuelche meridional. Mundo Ameghiniano, 7, 1-3.

Halvorsen, P. (2011). Identidades enmascaradas en la Patagonia. Uniones entre nativos y foráneos. Buenos Aires: Patagonia Sur libros.

Herrera, N. M. (1926). Mapa del Territorio de Santa Cruz.

Jefatura de Policía (1933-34). Plano del territorio de Santa Cruz.

Jones, O., \& Sullivan, C. (1989). The Parks Canada Glass Glossary for the description of containers, tableware, flat glass, and closures. Canada: National Historic Parks and Sites, Canadian Parks Service.

Kendrick, G. (1963). The Antique Bottle Collector. Nevada:
Western Printing \& Publishing Co.

Kendrick, G. (1967). Bottle Fragments betray age of Historical sites. El Palacio, 74(2), 19-24.

Kendrick, G. (1968). The Mouth-Blown Bottle. Ann Arbor: Edwards Brothers.

Lefrancois, A., \& Porri, P. (1927). Plano del territorio nacional de Santa Cruz.

Nuevo Delaunay, A. (2012). Disarticulation of Aónikenk huntergatherer lifeways during the late nineteenth and early twentieth centuries: two case studies from Argentinean Patagonia. Historical Archaeology, 46, 149-164.

Nuevo Delaunay, A. (2016). Transformación del paisaje arqueológico rural en el centro-oeste de la provincia de Santa Cruz, siglo XX. Tesis de Doctorado: Universidad de Buenos Aires.

Nuevo Delaunay, A., Belardi, J. B., Carballo Marina, F., Saletta, M. J., \& De Angelis, H. (2017). Glass and stoneware knapped tools among southern continental Patagonian and Fuegian hunter-gatherers from the late sixteenth to the twentieth century. Antiquity, 91(359), 13301343.

Priegue, N. C. (2007). En memoria de los abuelos. Historia de vida de Luisa Pascual. Bahía Blanca: Publitek.

Rock, J. (1990). Basic Bottle Identification. California: Klamath National Forest, U.S. Forest Service.

Rodríguez, M. E. (2008). Camusu Aike: de la visibilización en los archivos a la re-visibilización como comunidad. Bariloche: 3ras Jornadas de Historia de la Patagonia.

Rodríguez, M. E. (2009). Trayectorias de una recuperación en suspenso (ex Reserva Lago Viedma). Avá. Revista de Antropología, 14, 1-22.

Rodríguez, M. E. (2010). De la "extinción" a la autoafirmación: Procesos de visibilización de la comunidad Tehuelche Camusu Aike (provincia de Santa Cruz, Argentina). Tesis Doctoral inédita: Faculty of the Graduate School of Arts and Sciences of Georgetown University.

Rodríguez, M. E., \& Delrío, W. (2000). "Los tehuelches”, Un paseo etnohistórico. En C. J. Godoy Manríquez (Ed.), El gran libro de la Provincia de Santa Cruz (pp. 428460). Barcelona: Alfa-Milenio.

Rodríguez, M. E., \& Horlent, L. (2016). Tehuelches y selk'nam (Santa Cruz y Tierra del Fuego): no desaparecimos. En S. Hirsch \& A. Lazzari (Eds.), Pueblos indigenas en la Argentina: historias, culturas, lenguas y educación, fascículo 12 (pp. 5-40). Ciudad Autónoma de Buenos Aires: Ministerio de Educación y Deportes de la Nación.

Schiffer, M. B. (1990). Contexto arqueológico y contexto sistémico. Boletín de Antropología Americana, 22, 
81-93.

Skibo, J. M., \& Schiffer, M. B. (2001). Understanding artifact variability and change: A behavioral framework. En M. B. Schiffer (Ed.), Anthropological Perspectives on
Technology (pp. 133-149). Albuquerque: University of New Mexico Press.

Wilkinson \& Lefrancois, A. (1903). Plano de la Gobernación de Santa Cruz y Territorio de Magallanes. 